\title{
The effect of Hericium erinaceum on the prevention of chemically induced experimental colitis in rats
}

\author{
Ali Durmus ${ }^{1}$, Ilgim Durmus ${ }^{2}$, Omer Bender ${ }^{3}$, and Oguzhan Karatepe ${ }^{3}$
}

${ }^{1}$ Department of Surgery, Nisantasi University, Istanbul; ${ }^{2}$ Department of Medical Biotechnology, Acibadem University, Istanbul; ${ }^{3}$ Department of SHMYO (Health Occupation School), Yeni Yuzyil University, Istanbul, Turkey

\author{
Received: February 5, 2019 \\ Revised : September 4, 2019 \\ Accepted: December 18, 2019

\section{Correspondence to} \\ Ilgim Durmus, Ph.D. \\ Department of Medical \\ Biotechnology, Acibadem \\ University, İçerenköy Mahallesi, \\ Kayışdağ 1 Cd. No. 32, 34752, \\ Ataşehir/Istanbul, Turkey \\ Tel: +90-212-230-2008 \\ Fax: +90-212-230-4949 \\ E-mail: ilgim.durmus@gmail.com \\ https://orcid.org/0000-0003- \\ 3915-7315
}

Background/Aims: The aim of this study is to investigate the effects of the Hericium erinaceum on an experimental colitis model.

Methods: Twenty-four Wistar albino were included in this study. Rats were divided into three groups. Group $1(n=8)$ was sham group. Group 2 is the group of chemically induced by intrarectal administration of trinitrobenzene sulfonic acid (TNBS) resulting in colitis. Group $3(n=8)$ is the group that was treated 7 days before and 7 days after with $H$. erinaceum resulting in colitis. The activity of colitis was evaluated macroscopically and microscopically in rats. In other words, nitric oxide (NO) levels, malondialdehyde (MDA), interleukin 6 (IL-6), nuclear factor-kappa $\mathrm{B}(\mathrm{NF}-\kappa \mathrm{B})$ and, tumor necrosis factor- $\alpha$ (TNF- $\alpha)$ in addition to the myeloperoxidasem (MPO) activities was determined.

Results: The rate of TNBS-induced colitis caused to increase the level of MDA activities meaningfully in the colitis group than the control group. The results indicated that MDA ( $p=0.001)$, NO ( $p=0.001), \operatorname{IL}-6(p=0.001)$, MPO $(p=0.878)$, TNF- $\alpha(p=0.001)$, and NF- $\kappa$ B levels of treatment group decreased in the blood and colon tissues because of the $H$. erinaceum treatment when compared to the colitis group. H. erinaceum treatment was related to the declining of MDA, NF- $\kappa B$, NO, IL-6, and TNF- $\alpha$ levels.

Conclusions: $H$. erinaceum had a positive effect on the colitis by reducing oxidative damage in blood and tissue.

Keywords: Fungal proteins; Colitis; Rats; Colon

\section{INTRODUCTION}

Inflammatory bowel disease (IBD) is a chronic inflammatory disease of unknown cause, and effective treatment options have not been discovered [1]. Recent data increasingly indicate that signaling molecules and pathways are responsible for the onset and progression of the disease. There is evidence that the increased regulation of inflammatory mediators, such as cytokines, chemokines, and adhesion molecules, plays an essential role in both human and experimental colitis models $[2,3]$. Nuclear factor-kappa B (NF- $\mathrm{B}$ ) is responsible for the gene expression regulation of inflammatory mediators such as tumor necrosis factor- $\alpha$ (TNF- $\alpha$ ), interleukin 6 (IL-6), and nitric oxide synthase (iNOS) [4,5]. NF- $\mathrm{kB}$ is a key molecule involved in intestinal inflammation [6]. Studies that investigate the effect of anti-inflammatory drugs on the insula in IBD are difficult. For this reason, studies use animal models to investigate approaches to reduce inflammation and to activate various pathways. 
Hericium erinaceus (HE), known as lion's mane, is a medicinal mushroom and has been historical used in traditional Chinese medicine. HE mycelium and fruit bodies show great promise as substances that improve health such as brain and nerve health. The animal and in vitro experiments that investigated substances extracted from HE showed good results. In particular, the mycelium extract has been used clinically as an anti-inflammatory agent and in cytotoxic and cardiovascular antineoplastic research as an antibacterial and neuroprotective agent $[7,8]$. HE represents an important opportunity to treat Alzheimer's and Parkinson's diseases because HE is used as an anti-inflammatory medicine and promotes nerve growth factor gene expression.

The aim of this study was to evaluate the protective effect of HE treatment in a TNBS-induced colitis rat model.

\section{METHODS}

Twenty-four Wistar albino rats (225 to $275 \mathrm{~g}$ ) were included in this study. Rats were kept in wire-mesh bottomed cages under a 12-hour light/12-hour dark cycle at a constant room temperature $\left(22^{\circ} \mathrm{C} \pm 2{ }^{\circ} \mathrm{C}\right)$. A standard chow diet and water were provided. The study was approved by the Istanbul University ethics committee (number 2013-88).

\section{Chemical reagent}

2,4,6-Trinitrobenzene sulfonic acid (TNBS) was purchased from Sigma-Aldrich. The chemical formula of TNBS is $\mathrm{C}_{6} \mathrm{H}_{3} \mathrm{~N}_{3} \mathrm{O}_{9} \mathrm{~S}$. Its other names are picrylsulfonic acid and trinitrobenzene. It has extreme oxidative properties. HE plays an antioxidative role because of its active substances and reducing ability. Its ethanol and hot water extracts have very high antioxidant activity in vitro. HE was purchased from Health for Life Trading Co., Ltd. HE (250 and $500 \mathrm{mg} / \mathrm{kg} /$ day body weight) was administered orally to rats.

\section{Induction of colitis}

Rats were slightly anesthetized with ether after a 24hour fast. A 5-French polyurethane cannula was placed into the anus, and the tip was inserted to $8 \mathrm{~cm}$ proximal to the anal verge [9]. TNBS (10 mg/0.25 mL), which was dissolved in 50\% ethanol, was inoculated into the colon through the cannula to induce colitis. Then, the rats were headstand to observe complications. The rats with complications were excluded from this study, and a new experimental subject was included in this study.

Three groups with an equal number of animals were formed. Group 1 was the sham group $(n=8)$. After catheterization, the anesthetized rats were substituted with $0.9 \mathrm{~mL}$ of $0.8 \% \mathrm{NaCl}$ through the rectum. Group 2 was the colitis group $(n=8)$. To induce colitis, after catheterization through rectum, the mixture $35 \mathrm{mg} / 0.2 \mathrm{~mL}$ TNBS and $0.4 \mathrm{~mL} \mathrm{50 \%} \mathrm{ethanol)} \mathrm{was} \mathrm{administered} \mathrm{to} \mathrm{the}$ anesthetized rats. Group 3 was the treatment group $(\mathrm{n}=$ 8). After catheterization and colitis induction, $35 \mathrm{mg} / 0.2$ $\mathrm{mL}$ and $0.4 \mathrm{~mL} 50 \%$ of ethanol were administered to the anesthetized rats rectally administered TNBS. Additionally, the rats received $\mathrm{HE}$ (250 and $500 \mathrm{mg} / \mathrm{kg} /$ day body weight) orally 7 days before colitis induction and 7 days after colitis induction. The colon weight and colon length of each rat were recorded.

\section{Surgical procedure}

The surgical procedure under sterile conditions was applied to the experimental group on the 7 th day of treatment. Intramuscular administration of $70 \mathrm{mg} / \mathrm{kg}$ ketamine hydrochloride and $7 \mathrm{mg} / \mathrm{kg}$ xylazine was used to anesthetize all Wistar albino rats. General anesthesia and spontaneous respiration were used. Povidone iodine was used to clean the abdominal region. The part of the colon with induced colitis was transected distal to the rectum as low as possible, and a nearly $9 \mathrm{~cm}$ long colonic segment was collected. The segment was opened longitudinally, and $0.8 \%$ saline solution was used to rinse the feces. For the analysis of biochemical parameters, $4 \mathrm{~mL}$ blood was collected. For biochemical and histopathological research, tissue samples were collected and transferred to a $0.8 \% \mathrm{NaCl}$ solution or $9 \%$ formaldehyde solution. Blood samples were centrifuged and stored at $-80^{\circ} \mathrm{C}$ for biochemical research. Last, rats were sacrificed using a lethal dose of thiopental sodium.

\section{Histopathological evaluation}

\section{Macroscopic score}

Macroscopic changes in the colonic mucosa were categorized into five levels (Table 1).

\section{Microscopic score}

The colon was divided into two parts for pathologic 
Table 1. Levels of macroscopic changes in colonic mucosa

\begin{tabular}{ll}
\hline Score & \multicolumn{1}{c}{ Macroscopic changes } \\
\hline 0 & No colonic injury \\
\hline 1 & Hyperemia but not ulcer \\
2 & Linear ulcer but not colonic wall thickness \\
3 & Linear ulcer and colonic wall thickening at one spot \\
4 & Colonic ulcer at multiple spots \\
\hline 5 & Main ulcer and perforation \\
\hline
\end{tabular}

and biochemical studies after the macroscopic evaluation of the mucosa. The histopathological study was performed at Istanbul University. Colon tissue was separated into $5 \mathrm{~mm}$ sections and fixed in $9 \%$ formaldehyde [10]. After embedding in paraffin and staining with hematoxylin-eosin, $5 \mathrm{~mm}$ sections were prepared and evaluated with a light microscope. Inflammatory infiltration, thrombosis, apoptosis, necrosis, and vascular congestion were analyzed. All tests and microscopic alterations in the mucosa were evaluated from o to 3 by a histopathologist blinded to the groups.

Biochemical evaluation of the tissue and blood

For the biochemical assays, blood and terminal ileum were obtained. Blood samples were collected using a sterile needle by intracardiac puncture. Next, blood samples were obtained, centrifuged and separated into serum samples. Nitric oxide (NO), NF-кB, TNF-a, malondialdehyde (MDA), myeloperoxidasem (MPO), and IL- 6 were evaluated. Tissues were rinsed with serum, weighed and homogenized for biochemical investigation. Distal colon samples were collected for biochemical evaluation. To detect inflammation, IL-6, MPO and TNF-a were evaluated. To determine the rate of apoptosis, NO and MDA, which can indicate the extent of oxidative damage, were investigated.

\section{MPO measurement}

Tissues were homogenized, incubated in $0.5 \%$ hexadecyl-trimethylammonium bromide ( $\mathrm{pH} 5.5$ ) and $0.026 \%$ ortho-dianisidine dihydrochloride, and $0.018 \% \mathrm{H}_{2} \mathrm{O}_{2}$ was added. The reaction time was half an hour. The reaction was validated with sodium azide. All tests were performed twice and checked.
MDA measurement

MDA and thiobarbituric acid were examined by using a spectrophotometric procedure. Then, $0.2 \mathrm{~mL}$ of serum, $25 \mu \mathrm{L}$ of butylated hydroxytoluene ( $\mathrm{pH}$ 7.4) and $0.8 \mathrm{~mL}$ of tamponated phosphate were mixed. The prepared solution was mixed with $0.5 \mathrm{~mL}$ of $30 \%$ trichloroacetic hydro barbituric acid, put on ice for 2 hours and then centrifuged at $25^{\circ} \mathrm{C}$ for 15 minutes at 2,000 $\times g$. After centrifugation, ethylene diamine, tetra acetic thiobarbituric acid ( $0.070 \mathrm{mM})$ and $0.9 \%$ thiobarbituric acid ( $0.20 \mathrm{~mL}$ of $0.1 \mathrm{~mol} / \mathrm{L})$ was added to each $\mathrm{mL}$. The supernatant was reserved in boiled water and left to cool at room temperature for 15 minutes. Spectrometric measurement was performed for the last prepared supernatant at $532 \mathrm{~nm}$ wavelength. The outcomes were recorded as $\mathrm{nmol} / \mathrm{mL}$.

\section{NO measurement}

The serum NO level was analyzed with Griess reagent. For the first phase, nitrate reductase was used to degrade the nitrate in the serum. For the second phase, nitrogen purple was improved by Griess reagent. Zinc sulfate was added to this mixture and centrifuged at $10,000 \times \mathrm{g}$ for 4 minutes. The measurement was performed using azo dye by chromatographic spectrometer. The outcomes were recorded as $\mathrm{nmol} / \mathrm{mL}$.

TNF- $\alpha$ and IL-6 measurements

An enzyme-linked immunosorbent assay was used to measure TNF- $\alpha$ and IL-6. The lowest values that could be determined were $0.12 \mathrm{pg} / \mathrm{mL}$ and $0.03 \mathrm{pg} / \mathrm{mL}$ for TNF- $\alpha$ and IL-6, respectively.

NF-KB measurement

The colon of rats was embedded in 10\% buffered for- 
malin. Paraffin blocks were cut into $5 \mu \mathrm{m}$ slices. The paraffin-embedded specimens were deparaffinized and postfixed in 100\% acetone for 5 minutes to block endogenous peroxidase activity. Antigen retrieval was performed, and the sections were incubated with primary rabbit polyclonal anti-NF- $\kappa \mathrm{B}$ antibody (anti-rabbit $\mathrm{P}_{50 \mathrm{Ab}-2)}$ for 30 minutes at room temperature. Then, the slides were treated with biotinylated secondary antibody and streptavidin-conjugated horseradish peroxidase. Hematoxylin was used to stain for visualization [11].

\section{Statistical evaluation}

The results were evaluated as the mean and standard deviation. All data are expressed as the mean \pm SD. Kruskal-Wallis and analysis of variance were used for statistical analysis, and $p<0.05$ was accepted as significant (SPSS version 25.0 for Windows, IBM Co., Armonk, NY, USA).

\section{RESULT}

\section{Macroscopic colitis score}

The macroscopic score was markedly increased in all rats administered TNBS compared with the rats in the sham group $(p<0.001)$. Macroscopic injury of the colon on the 7 th day after TNBS administration indicated hyperemia, ulceration, colonic wall thickness and severe adhesions between the colon and other organs. HE treatment markedly reduced the macroscopic score in TNBS-treated animals $(p<0.001)$.

To investigate colitis formation, the length of the colon and the weight of the colon in the sham, HE treatment and nontreatment groups were compared. In the HE-treated group, the length of the colon was similar to that of the sham group, and the weight of the colon was similar to that of the sham group. Colon weight was increased, and colon length was decreased in the untreated group (Fig. 1).

\section{Microscopic colitis score}

The microscopic score was markedly higher in all rats administered TNBS than in the rats in the sham group $(p<0.001$ ) (Fig. 2). After treatment with HE and TNBS, the pathologic score decreased markedly $(p<0.001)$. Fig. 3 shows that HE administration resulted in less muco-

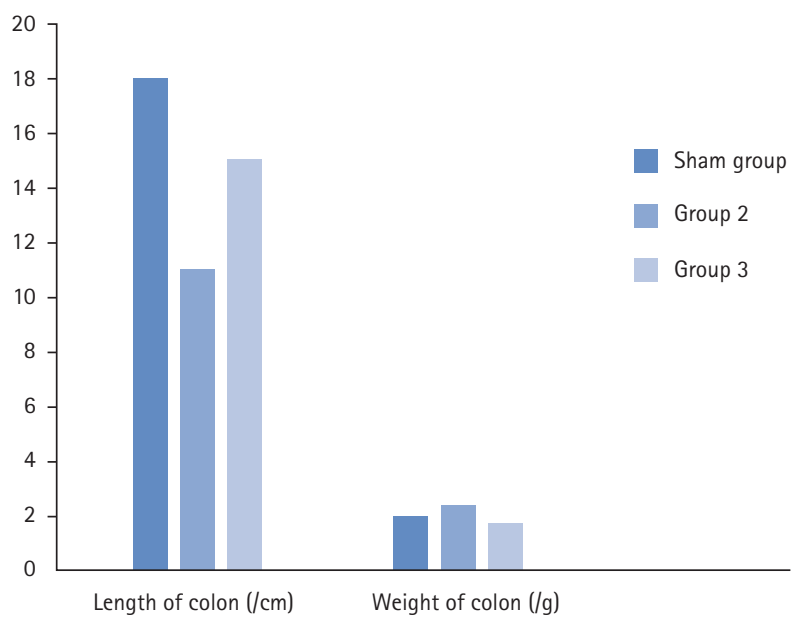

Figure 1. Graph of colon length and weight for sham group, group 2, and group 3. The sham group, the treatment and nontreatment groups were compared to evaluate colitis formation. In the group 3, the colon length and the weight was close to sham group whereas, the length of colon was decreased and the weight of colon increased for the group 2.

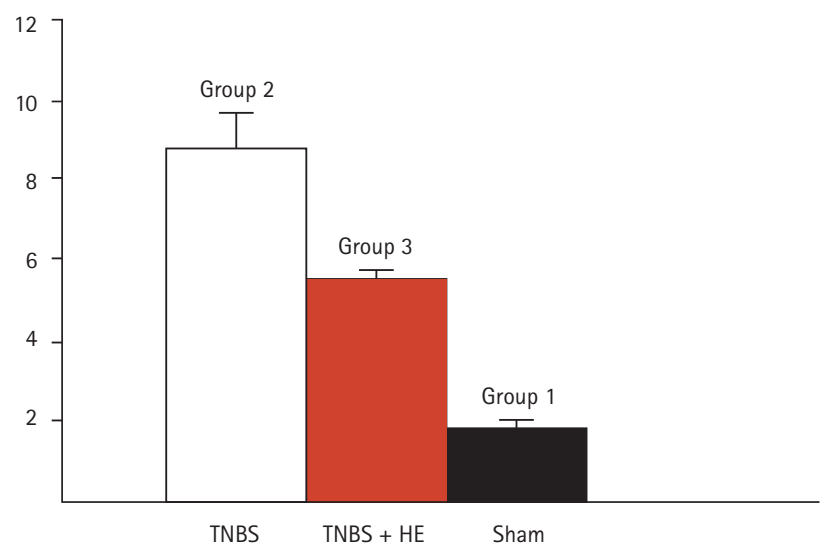

Figure 2. Microscopic colitis score. The significance between 1 and 2 and 3 is $p<0.05 ; 3$ and 2 is $p<0.05$. TNBS, trinitrobenzene sulfonic acid; HE, Hericium erinaceus.

sal injury, healed mucosal structure, and epithelial integrity. According to immunohistochemistry analysis, NF- $\kappa \mathrm{B}$ expression was higher in the rats from group 2 higher than in rats from group 3.

\section{Biochemical results}

The colonic MDA levels are shown in Fig. 4. The oxidative damage parameters (NO and MDA levels) were compared among the groups. The level of the sham group was markedly different from that of the treatment group 
Table 2. Colonic tissue, inflammatory, and oxidative damage parameters

\begin{tabular}{lcccc}
\hline Measure article & Sham group & Group 2 & Group 3 & $p$ value \\
\hline TNF- $\alpha$ & $0.243 \pm 0.027$ & $0.675 \pm 0.042$ & $0.276 \pm 0.038$ & 0.001 \\
IL-6 & $0.191 \pm 0.053$ & $0.635 \pm 0.084$ & $0.248 \pm 0.061$ & 0.001 \\
MDA & $1.089 \pm 0.115$ & $3.462 \pm 0.292$ & $1.167 \pm 0.158$ & 0.001 \\
NO & $119.000 \pm 6.120$ & $144.300 \pm 8.315$ & $121.000 \pm 6.830$ & 0.001 \\
MPO & $0.385 \pm 0.132$ & $0.330 \pm 0.121$ & $0.420 \pm 0.147$ & 0.878 \\
\hline
\end{tabular}

All group was compared to each other and $p$ value for group 2 and group 3 was given in the last column.

TNF- $\alpha$, tumor necrosis factor- $\alpha$; IL-6, interleukin 6; MDA, malondialdehyde; NO, nitric oxide; MPO, myelope roxidasem.

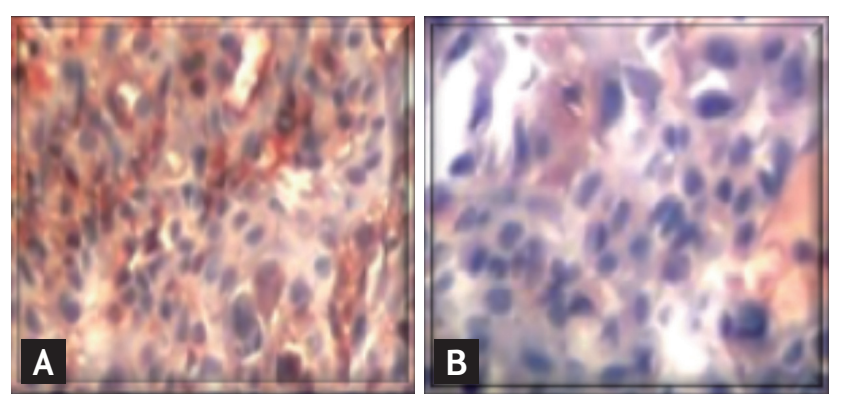

Figure 3. Comparison of the inflammation between group 2 and group 3 (H\&E stain, $\times 400)$. (A) Up-regulation of the nuclear factor-kappa $\mathrm{B}(\mathrm{NF}-\kappa \mathrm{B})$ expression in the colon in the trinitrobenzene sulfonic acid-induced colitis. (B) Colon from the Hericium erinacues treated rats. There was a minimum NF- $\mathrm{B}$ expression.

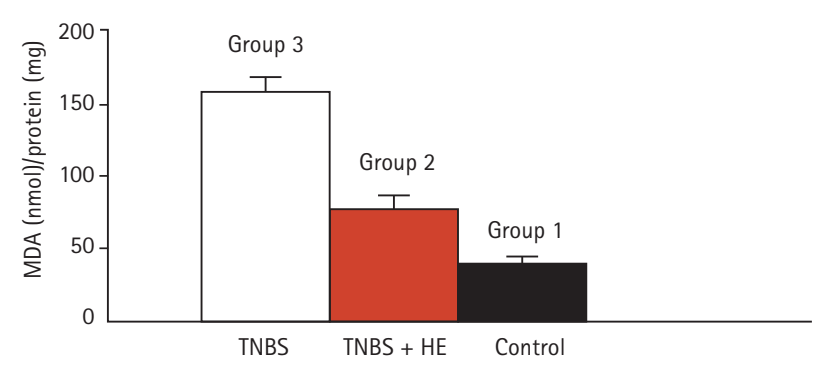

Figure 4. Colonic malondialdehyde (MDA) levels of all groups. Values with different letters have significance according to analysis of variance test. The significance between 2 and 3 is $p<0.001$. TNBS, trinitrobenzene sulfonic acid; HE, Hericium erinaceus.

and colitis group. The blood and tissue levels of NO and MDA were markedly decreased in the HE-treated group compared with the colitis group (blood NO, $p=$ o.oo1; tissue NO, $p=0.001$; blood MDA, $p=0.001$; tissue
MDA, $p=0.001$ ). The comparison of oxidative damage is shown in Table 2.

IL-6 and TNF- $\alpha$ levels in blood and tissue were analyzed as inflammatory parameters. The levels in the treatment group and colitis group results were markedly different from the levels in group $1(p=0.001)$. Compared with the colitis group, group 3 showed substantially decreased levels. Another parameter of inflammation in tissue is MPO level. The MPO levels of the treatment and colitis groups were not significantly different $(p=$ o.878). The inflammatory damage results are shown in Table 2.

The level of NF- $\kappa \mathrm{B}$ expression in the untreated group was high $(p<0.01)$. The NF- $\kappa B$ expression level was lower in the HE treatment group than in the TNBS-induced colitis model (Fig. 3).

\section{DISCUSSION}

HE is an edible and medicinal mushroom and has also been used as a traditional Chinese medicine. Various studies have shown that the content of the HE is useful for medical purposes because of its high protein, low fat and high nutritional value [12,13]. HE contains erinaceus hericenone, $\mathrm{CH}[14,15]$, erinacines AK $[16,17]$, orcinol derivatives (mycelium) [18], sialic acid-binding lectin [19], ergosterol and beta-sitosterol [20]. Since hericenone and erinacine strongly stimulate nerve growth factor synthesis, which allows for neuron repair and regeneration, HE increases nerve growth factor synthesis, and its compound activity slows neuronal cell death [17]. For this reason, HE can be used as a nutritional therapy 
in the treatment of dementia, Alzheimer's disease and Parkinson's disease. The orcinol derivatives obtained from the mycelium of HE show antibacterial and antifungal activity against certain pathogenic microorganisms (such as Bacillus subtilis and Saccharomyces cerevisiae) [21]. Another component of HE, sialic acid-binding lectin, can be used to identify, quantify, localize, purify, and characterize many biomolecules (such as glycoconjugates). Sialic acid plays an important role at the protein and cell levels in the biological recognition mechanisms, and lectins can be used as specific probes for sialic acid species, which act as molecular markers in pathological and physiological development [22]. Ergosterol is a major fungal sterol, and beta-sitosterol reduces the absorption of cholesterol in the intestinal lumen. In the study by Mbambo et al. [23], these stressors were shown to have antifungal activity. HE also has a prebiotic effect; it contained the polysaccharide $\beta$-glucan and the monosaccharides mannose (approximately $55.70 \%$ ), arabinose (approximately 0.7\%), rhamnose (approximately 3.75\%), xylose (approximately 1.60\%), galactose (approximately 5.12\%), and glucose (approximately 33.10\%) [24]. Since HE contains physiologically important compounds, there are some studies on gastritis and gastrointestinal infections. Extracts of HE mycelium have been indicated to inhibit gastric mucosal damage and improve gastric ulcers [25], with the inclusion of $\mathrm{HE}$ in the diet improved colonic health and IBD, and HE has anti-inflammatory activity $[26,27]$.

The results of our study suggest that treatment with $\mathrm{HE}$ has a positive impact on colitis in TNBS-induced rats by reducing oxidative damage in blood and tissue. This is indicated by the significantly decreased NF- $\kappa \mathrm{B}$, MDA, NO, IL6, and TNF- $\alpha$ levels in blood and tissue. Additionally, the current study shows macroscopic and microscopic colitis scores that indicate less mucosal damage and improved mucosal structure.

IBD is a chronic, recurrent disease characterized by idiopathic inflammation of the gastrointestinal tract whose pathogenesis is not known precisely. IBD mainly consists of two clinical forms, Crohn's disease (CD) and ulcerative colitis (UC). UC is characterized by recurrent weakness of the mucosal layer of the colon [28]. It is most commonly seen in the rectum, spreading to other parts of the colon with continuity. CD is a disease of the digestive tract that may be anywhere from oral to annu- lar, with focal, asymmetric, or transmural involvement [29]. Some clinical studies have shown that genetic factors increase the risk of developing IBD [30]. The effects of environmental factors on the formation of IBD and processes are not fully understood. Clinical and epidemiological studies indicate that this disease can develop in genetically susceptible individuals after exposure to different antigens or environmental factors. Exacerbations and healing may be associated with these factors. According to our results, $\mathrm{HE}$ can be a protective agent for IBD.

Immune factors play an effective role in the pathogenesis of IBD. Some cytokines are proinflammatory (IL-1, IL-2, IL-6, and TNF- $\alpha$ ), and some are anti-inflammatory (IL-8, IL-10, IL-11, and TNF- $\gamma$ ). HE treatment improved the inflammatory response of rats because treatment of TNBS-induced rats with HE resulted in increased levels of some anti-inflammatory cytokines compared to the untreated group [24]. Our study also showed that feeding TNBS-treated rats HE had a good inflammatory effect because the TNF- $\alpha$ and IL-6 levels of HE-fed rats were lower than those of the control group. It was also demonstrated that the administration of HE decreased inflammation, MDA levels and MPO activity in rats with TNBS-induced colitis.

Several compounds that initiate the immune response in the digestive tract have been identified, and experimental models, in which these compounds are generated, have been used to understand the pathogenesis of the disease and to develop drug treatments. TNBS is one of these compounds, and the disease model created by this substance is widely used in experimental studies since it produces effects similar to human UC [31,32]. In this study, cytokines such as TNF- $\alpha$, MPO, NO, MDA, and IL- 6 were investigated to determine the cellular activities of the TNBS-induced colitis model because cytokines are key signaling molecules of the intestinal immune system that play an important role in IBD. In vivo and in vitro studies indicate that the levels of proinflammatory cytokines such as TNF- $\alpha$ and IL- 6 are elevated in IBD and that the severity of inflammation is directly proportional to the levels of these cytokines [33]. Moreover, the macroscopic colitis score of this study showed that colonic wall thickness, ulcers, severe adhesions between the colon and other organ hyperemia were observed on the 7 th day in TNBS-induced rats. 
The comparison of the microscopic colitis scores of the TNBS-induced group and HE treatment group showed that the HE treatment group had less mucosal injury, improved mucosal structure, and epithelial integrity. In one study, the HE-treated group showed more pronounced improvement than did the TNBS-induced group. Mucosal erosion and ulcers were observed in the TNBS-induced group, and less injury was observed in the HE group than in the TNBS group. Our results clearly demonstrated that the HE extracts could promote the protection of the gastric mucosa and give positive results in the experimental models of IBD.

One previous study showed that HE extracts contributed to the growth of beneficial gut bacteria and ameliorated host immunity in an experimental IBD model. When TNBS-induced rats were treated with HE extracts, the expression levels of inflammatory factors were changed. MPO levels declined, and TNF- $\alpha$ levels in the peripheral blood and colon of the normal group were lower than those in TNBS-treated rats. In our study, according to the biochemical results, there was a decrease in MDA, NO, IL6, and TNF- $\alpha$ levels in the HE treatment group compared with the TNBS-induced colitis group. Treatment with HE enhanced all the inflammatory responses that we investigated.

Excess TNF- $\alpha$ expression damages epithelial barriers and induces apoptosis in epithelial cells [34]. In accordance with the literature, TNF-a expression was more intense in the areas of inflammation and ulceration in the colon mucosa of the colitis group. Zhou et al. [35] showed that TNF-a expression was increased in a TNBS-induced colitis model. This study showed that HE treatment decreased TNF- $\alpha$ and IL- 6 levels because the TNF- $\alpha$ and IL-6 levels in the TNBS-induced colitis model were significantly higher than those in the HE group.

One of the previous observations showed that MPO levels in the HE group were lower than those in the colitis group, and HE extracts decreased colon inflammation in rats with IBD [22]. When MPO activity was examined, the values obtained from the colitis group were considerably higher than those of the control group $(p$ $<0,05)$, and inflammation was observed. The MPO levels measured at the tissue level were significantly lower in the treatment groups than those in the colitis group $(p<0.05)$. Furthermore, the MDA level of the colonic tissues was found to be increased in the TNBS-treated group compared to the sham group. Treatment with HE caused a significant decrease in the MDA level in TNBS-induced colitis rats.

$\mathrm{NF}-\mathrm{\kappa B}$ proteins are transcription factors that control apoptosis, cell cycle progression, cell growth, and differentiation. The NF- $\kappa \mathrm{B}$ pathway is involved in colonic inflammation, and increased NF- $\kappa \mathrm{B}$ protein expression has been observed in the mucosa of patients with unspecific colitis, UC, and CD [36]. Proinflammatory cytokines induce NF- $\mathrm{KB}$ activation in different cell types, and this could be initiated by oxidative stress, which has a significant role in the pathogenesis and progression of IBD. Our biochemical findings also indicate that NF- $\mathrm{BB}$ expression decreased with $\mathrm{HE}$ administration, whereas NF- $\kappa B$ overexpression was observed in TNBS-induced colitis. The inhibition of NF-kB activation could be a possible treatment strategy for IBD.

As a limitation of this study, we analyzed MDA, NO, TNF- $\alpha$, MPO, and NF- $\kappa B$ values to measure oxidative damage, which is similar to some studies [5,35,37]. The antioxidant effects of HE, the total antioxidant concentration and the protein expression levels of heme oxygenase-1 (HO-1) and NAD(P)H:quinone oxidoreductase (NQO1). were not analyzed in this study so that there was not much data complexity during the experimental phase and to ensure that the costs of the experiment did not increase. In future studies by our group, these values will be examined.

In conclusion, oral HE treatment showed good efficacy in the experimental colitis model. Biochemical indexes and microscopic and macroscopic colitis scores were analyzed. TNF- $\alpha$, MPO, NO, MDA, and IL6 cytokines, which affect TBNS-induced colitis models, were examined, and TNF- $\alpha$, MPO, NO, MDA, and IL6 levels were lower in the HE treatment group than in the colitis groups. Less mucosal injury was detected in the HE treatment group than in the colitis group. In light of these results, HE has clinical potential for the improvement of IBD because HE has an anti-inflammatory properties. The main disadvantage of oral therapy with HE is the unknown dose required for the anti-inflammation effect. For this reason, this issue will be investigated in future studies. 


\section{KEY MESSAGE}

1. Hericium erinaceum had a positive effect on the colitis by reducing oxidative damage in blood and tissue.

2. Hericium erinaceum can be used as a nutritional treatment for inflammatory bowel disease patients

3. The long-term effect used of Hericium erinaceum should be studied in more detailed.

\section{Conflict of interest}

No potential conflict of interest relevant to this article was reported.

\section{Acknowledgments}

I would like thank Dr. Serap Durmus for help with the manuscript, data correction and biochemical results.

\section{REFERENCES}

1. Fakhoury M, Negrulj R, Mooranian A, Al-Salami H. Inflammatory bowel disease: clinical aspects and treatments. J Inflamm Res 2014;7:113-120.

2. Ajuebor MN, Swain MG. Role of chemokines and chemokine receptors in the gastrointestinal tract. Immunology 2002;105:137-143.

3. Matricon J, Barnich N, Ardid D. Immunopathogenesis of inflammatory bowel disease. Self Nonself 2010;1:299-309.

4. Garcia-Mediavilla V, Crespo I, Collado PS, et al. The anti-inflammatory flavones quercetin and kaempferol cause inhibition of inducible nitric oxide synthase, cyclooxygenase-2 and reactive C-protein, and down-regulation of the nuclear factor kappaB pathway in Chang Liver cells. Eur J Pharmacol 2007;557:221-229.

5. Soufli I, Toumi R, Rafa H, Touil-Boukoffa C. Overview of cytokines and nitric oxide involvement in immuno-pathogenesis of inflammatory bowel diseases. World J Gastrointest Pharmacol Ther 2016;7:353-360.

6. Elson CO, Sartor RB, Tennyson GS, Riddell RH. Experimental models of inflammatory bowel disease. Gastroenterology 1995;109:1344-1367.

7. Kuo HC, Lu CC, Shen $\mathrm{CH}$, et al. Hericium erinaceus mycelium and its isolated erinacine A protection from
MPTP-induced neurotoxicity through the ER stress, triggering an apoptosis cascade. J Transl Med 2016;14:78.

8. Mizuno T, Wasa T, Ito H, Suzuki C, Ukai N. Antitumor-active polysaccharides isolated from the fruiting body of Hericium erinaceum, an edible and medicinal mushroom called yamabushitake or houtou. Biosci Biotechnol Biochem 1992;56:347-348.

9. Karatepe O, Altiok M, Battal M, et al. The effect of progesterone in the prevention of the chemically induced experimental colitis in rats. Acta Cir Bras 2012;27:23-29.

10. Yamamoto M, Yoshizaki K, Kishimoto T, Ito H. IL-6 is required for the development of Th1 cell-mediated murine colitis. J Immunol 2000;164:4878-4882.

11. Khater DS, Kasem RF, Morsy RA. Immunohistochemical analysis of $\mathrm{Nf}-\kappa \mathrm{B}$ expression and its relation to apoptosis and proliferation in different odontogenic tumors. Int $\mathrm{J}$ Cancer Res 2017;13:76-83.

12. Rossi P, Cesaroni V, Brandalise F, et al. Dietary supplementation of lion's mane medicinal mushroom, hericium erinaceus (agaricomycetes), and spatial memory in wild-type mice. Int J Med Mushrooms 2018;20:485-494.

13. Powell M. Medicinal Mushrooms: A Clinical Guide. East Sussex (UK): Mycology Press, 2010.

14. Mori K, Obara Y, Hirota M, et al. Nerve growth factor-inducing activity of Hericium erinaceus in $1321 \mathrm{~N} 1$ human astrocytoma cells. Biol Pharm Bull 2008;31:1727-1732.

15. Yaoita Y, Danbara K, Kikuchi M. Two new aromatic compounds from Hericium erinaceum (BULL.: FR.) PERS(1). Chem Pharm Bull (Tokyo) 2005;53:1202-1203.

16. Chiu CH, Chyau CC, Chen CC, et al. Erinacine a-enriched hericium erinaceus mycelium produces antidepressant-like effects through modulating BDNF/PI $3 \mathrm{~K} / \mathrm{Akt} /$ GSK-3 $\beta$ signaling in mice. Int J Mol Sci 2018;19.

17. Li IC, Lee LY, Tzeng TT, et al. Neurohealth properties of hericium erinaceus mycelia enriched with erinacines. Behav Neurol 2018;2018:5802634.

18. Okamoto K, Sakai T, Shimada A, et al. Antimicrobial chlorinated orcinol derivatives from mycelia of Hericium erinaceum. Phytochemistry 1993;34:1445-1446.

19. Kawagishi H, Mori H, Uno A, Kimura A, Chiba S. A sialic acid-binding lectin from the mushroom Hericium erinaceum. FEBS Lett 1994;340:56-58.

20. Li JL, Lu L, Dai CC, Chen K, Qiu JY. A comparative study on sterols of ethanol extract and water extract from Hericium erinaceus. Zhongguo Zhong Yao Za Zhi 2001;26:831834 . 
21. Wong KH, Sabaratnam V, Abdullah N, Kuppusamy UR, Naidu M. Effects of cultivation techniques and processing on antimicrobial and antioxidant activities of Hericium erinaceus (Bull.:Fr.) pers. extracts. Food Technol Biotechnol 2009;47:47-55.

22. Lehmann F, Tiralongo E, Tiralongo J. Sialic acid-specific lectins: occurrence, specificity and function. Cell Mol Life Sci 2006;63:1331-1354.

23. Mbambo B, Odhav B, Mohanlall V. Antifungal activity of stigmasterol, sitosterol and ergosterol from Bulbine natalensis aker (Asphodelaceae). J Med Plant Res 2012;6:5135-5141.

24. Diling C, Xin Y, Chaoqun Z, et al. Extracts from Hericium erinaceus relieve inflammatory bowel disease by regulating immunity and gut microbiota. Oncotarget 2017;8:85838-85857.

25. He X, Wang X, Fang J, et al. Structures, biological activities, and industrial applications of the polysaccharides from Hericium erinaceus (Lion's Mane) mushroom: a review. Int J Biol Macromol 2017;97:228-237.

26. Wang XY, Yin JY, Nie SP, Xie MY. Isolation, purification and physicochemical properties of polysaccharide from fruiting body of Hericium erinaceus and its effect on colonic health of mice. Int J Biol Macromol 2018;107:13101319.

27. Qin M, Geng Y, Lu Z, et al. Anti-inflammatory effects of ethanol extract of lion's mane medicinal mushroom, hericium erinaceus (agaricomycetes), in mice with ulcerative colitis. Int J Med Mushrooms 2016;18:227-234.

28. Navaneethan U, Shen B. Pros and cons of medical man- agement of ulcerative colitis. Clin Colon Rectal Surg 2010;23:227-238.

29. Hanauer SB. Biologics in peri-operative management of Crohn's disease. Acta Gastroenterol Belg 2001;64:191-192.

30. Duerr RH. The genetics of inflammatory bowel disease. Gastroenterol Clin North Am 2002;31:63-76.

31. Low D, Nguyen DD, Mizoguchi E. Animal models of ulcerative colitis and their application in drug research. Drug Des Devel Ther 2013;7:1341-1357.

32. Blumberg RS, Saubermann LJ, Strober W. Animal models of mucosal inflammation and their relation to human inflammatory bowel disease. Curr Opin Immunol 1999;11:648-656.

33. Strober W, Fuss IJ. Proinflammatory cytokines in the pathogenesis of inflammatory bowel diseases. Gastroenterology 2011;140:1756-1767.

34. Yang $M$, Lin HB, Gong $S$, et al. Effect of Astragalus polysaccharides on expression of TNF- $\alpha$, IL-1 $\beta$ and NFATc 4 in a rat model of experimental colitis. Cytokine 2014;70:8186.

35. Zhou YH, Yu JP, Liu YF, et al. Effects of Ginkgo biloba extract on inflammatory mediators (SOD, MDA, TNF-alpha, NF-kappaBp65, IL-6) in TNBS-induced colitis in rats. Mediators Inflamm 2006;2006:92642.

36. Viennois E, Chen F, Merlin D. NF-אB pathway in colitis-associated cancers. Transl Gastrointest Cancer 2013;2:21-29.

37. Mammadov R, Suleyman B, Akturan S, et al. Effect of lutein on methotrexate-induced oxidative lung damage in rats: a biochemical and histopathological assessment. Korean J Intern Med 2019;34:1279-1286. 